\title{
Determinantes da Transferência de Tecnologia na Agroindústria Brasileira de Alimentos: Identificação e Caracterização'
}

\author{
André Yves Cribb²
}

\begin{abstract}
This article aims to identify and characterize determinants of technology transfer, from the perspective of agro-industrial firms operating in Brazil, and under the light of the core theoretical concepts. By reaching this objective, the article provides a basis for studies and practices relating to technological change in the agrifood sector. Particularly, it may help in defining or reformulating public policies favorable for the generation and application of technologies in the Brazilian food industry.
\end{abstract}

Key words: Agrifood sector; technological management; research organization.

\section{Resumo}

Este artigo objetiva identificar e caracterizar determinantes da transferência de tecnologia à luz dos conceitos teóricos básicos e na ótica de empresas agroindustriais que atuam no Brasil. Ao alcançar este objetivo, o artigo apresenta uma base para estudos e práticas referentes à mudança tecnológica no setor agroalimentar. Particularmente, ele pode auxiliar na definição ou reformulação de políticas públicas favoráveis para a geração e aplicação de tecnologias na agroindústria brasileira de alimentos.

Palavras-chave: Setor agroalimentar; gestão tecnológica; organização de pesquisa.

\footnotetext{
I O projeto de pesquisa do qual o artigo se originou foi executado graças ao suporte financeiro do CNPq - Conselho Nacional de Desenvolvimento Científico e Tecnológico (Cribb, 2000).

2 Pesquisador. Embrapa Agroindústria de Alimentos. Empresa Brasileira de Pesquisa Agropecuária. Av. das Américas, 2950I - Guaratiba, CEP: 23020-470, Rio de Janeiro - RJ, Brasil. Phone: +55 (2I) 3622-9744. E-mail: aycribb@ctaa.embrapa.br.
}

ISSN: 07I8-2724. (http://www.jotmi.org)

Journal of Technology Management \& Innovation (C) Universidad Alberto Hurtado, Facultad de Economía y Negocios 


\section{Introdução}

Nos últimos 35 anos, o agronegócio brasileiro tem-se comportado como um sistema produtivo altamente eficiente e competitivo. Tal eficiência e competitividade têm decorrido da elevação da produtividade total dos fatores (PTF) que, por exemplo, cresceu à taxa anual média de 3,30\% no período de 1975-2002. Esta taxa foi bem maior à dos Estados Unidos de América (EUA) ${ }^{3}$, a maior potência mundial agrícola no mesmo período (Gasques et al., 2004; Barros \& Barros, 2005; Jank et al., 2005; RIPA, 2008).

agronegócio tem participado expressivamente no valor do PIB - Produto Interno Bruto (Nunes \& Contini, 2000; Pinto, 2006) e no ajuste da balança comercial do Brasil (Jank \& Nassar, 2000; Pinto, 2006). Segundo RIPA (2008), o agronegócio brasileiro respondeu, em 2006, por $26,7 \%$ do PIB, 36\% dos empregos e 39,7\% das exportações. Entre 2000 e 2006, o setor registrou expansão de 15\%. O desempenho do agronegócio brasileiro destacou-se notadamente na agroindústria de alimentos, que foi objeto de várias iniciativas de inovação tecnológica e organizacional (Cribb, 2004).

A agroindústria de alimentos é tradicionalmente definida como "o conjunto das empresas que transformam produtos geralmente de origem agrícola para satisfazer as necessidades alimentares dos consumidores" (Malassis, 1979: 12, apud Nefussi, 1989). Austin (1981) acrescenta que a abrangência das operações de transformação pode ser ampla, incluindo a lavagem, triagem, mistura, cocção e processamento químico de alimentos provenientes da agricultura, fruticultura e pecuária. Nefussi (1989) explica que as empresas agroindustriais compram suas matériasprimas no segmento agropecuário e fabricam produtos alimentícios para ser comercializados, distribuídos e consumidos no mercado nacional e internacional. De acordo com Goodman e colaboradores (1990: 69), “o moderno sistema de alimentos se estende desde a agricultura, num extremo, passando pelo processamento, pela distribuição e pela venda no varejo, até o setor produtor de alimentos e refeições industrializadas (catering industry) e a preparação doméstica de alimentos, no outro".

\footnotetext{
${ }^{3}$ Nesse período, a PTF cresceu à taxa anual de I,57\% nos EUA.
}

A partir dos anos de 1990, a agroindustrialização foi relativamente rápida e intensa no Brasil. Segundo Wilkinson (1995 apud Reardon \& Barrett, 2000), ela é caracterizada por três conjuntos de mudanças: I) o crescimento de atividades de aquisição de matérias-primas agrícolas, distribuição e agroprocessamento, empreendido por grandes, médias e pequenas empresas agroindustriais atuando no Brasil; 2) mudança institucional e organizacional na relação entre empresas agroindustriais e estabelecimentos agrícolas, como crescimento da coordenação vertical; e; 3) mudanças concomitantes na agropecuária, tais como mudanças na composição do produto, tecnologia e estruturas setoriais e de mercado.

As potencialidades do mercado agroalimentar brasileiro tem atraído novos investimentos de companhias multinacionais. Aquisições têm constituído a estratégia mais comum de expansão e inserção em novos mercados. Os contratos, adotados entre as agroindústrias de alimentos e os agricultores, têm estabelecido preços diferentes, principalmente em função de requisitos de qualidade e práticas de cultivo, colheita e pós-colheita. Ao mesmo tempo, tem-se manifestado a aceitação do consumidor de alimentos a pagar preços premium. Frente a tais mudanças, os pequenos e médios produtores têm recorrido à ação coletiva para poder sobreviver e competir com as grandes empresas agroindustriais (Farina, 2001; Cribb e colaboradores, 2008).

No Brasil, a atuação das empresas agroindustriais tem ocorrido num contexto internacional caracterizado pela abertura comercial. Este contexto não só tem trazido novas oportunidades de mercado no exterior, mas também tem facilitado a presença de competidores estrangeiros no país (Cribb, 2004; RIPA, 2008). Em razão da importância da tecnologia no crescimento econômico (Rosenberg, 1982; Guellec, 1993; Guan et al., 2006; Wang \& Chien, 2007), as empresas agroindustriais têm-se encontrado, portanto, numa posição de permanente busca por recursos tecnológicos necessários ao enfrentamento dos desafios da competição internacional. Entretanto, no sistema agroalimentar, são relativamente poucas aquelas que têm estratégias próprias de atuação englobando ao mesmo tempo atividades de geração e aplicação de tecnologias (Arrow, 1962; Lin, 2003). Frente a essa realidade, existem no Brasil, como em outros países, organizações públicas de pesquisa e desenvolvimento 
(P\&D) para assistir cientifica e tecnologicamente as empresas agroindustriais.

O objetivo deste artigo é identificar e caracterizar determinantes da transferência de tecnologias de uma organização pública de pesquisa - denominada Embrapa Agroindústria de Alimentos ${ }^{4}$ - para as médias, pequenas e micro empresas agroindustriais que atuam no Brasil. $\mathrm{Na}$ sua segunda parte, $\mathrm{o}$ artigo apresenta as dimensões e características da transferência de tecnologia vista como processo multidimensional e interativo. $\mathrm{Na}$ sua terceira parte, define a metodologia da pesquisa, abrangendo as abordagens e os procedimentos utilizados na revisão de literatura, coleta de dados primários e observação direta em campo. Na sua quarta parte, descreve e discute os resultados da pesquisa. Na sua quinta parte, conclui com as principais contribuições e implicações da pesquisa.

\section{Multidimensionalidade e interatividade do processo de transferência de tecnologia}

A tecnologia pode ser definida como o conhecimento teórico e prático, relativo a certos tipos de ocorrências e atividades associadas à produção e transformação de materiais (Rosenberg, 1982; Burgelman et al, 2004). Importante na determinação da vantagem competitiva (UNCTAD, 1996), ela é apontada como ingrediente essencial no desempenho das empresas. Segundo Gilbert e Cordey-Hayes (1996), a chave de sucesso para uma organização ou empresa está incorporada na sua habilidade de implementar, dominar e valorizar conhecimentos tecnológicos. Nesse mesmo sentido, Burgelman e colaboradores (2004: 2) ressaltam que "a tecnologia é um recurso de alta relevância a diversas organizações; gerenciar esse recurso para vantagem competitiva significa integrá-lo na estratégia da empresa". Nesse sentido, a competitividade organizacional exige amplamente não apenas o conhecimento tecnológico mas também 0 gerenciamento tecnológico.

O conhecimento tecnológico refere-se basicamente "à competência de reconhecer problemas técnicos, desenvolver

\footnotetext{
${ }^{4}$ A Embrapa Agroindústria de Alimentos é uma unidade da Empresa Brasileira de Pesquisa Agropecuária. Ela tem por missão viabilizar soluções tecnológicas para a sustentabilidade da agroindústria de alimentos, com foco na inovação e atendendo às expectativas dos consumidores por qualidade e segurança (Embrapa Agroindústria de Alimentos, 2009).
}

novos conceitos e soluções tangíveis a problemas técnicos, (...) assim como à competência de aproveitar os conceitos e soluções tangíveis de maneira efetiva" (Autio \& Laamanen, 1995: 647). É embutido em pessoas, materiais, processos cognitivos e físicos, plantas, máquinas e ferramentas (Dosi, 1988; Lin, 2003; Burgelman et al., 2004).

O gerenciamento tecnológico é um processo complexo que consiste essencialmente na geração e aplicação de conhecimentos para desenvolver produtos e serviços assim como sistemas de produção e distribuição. Ele pode resultar na mudança tecnológica ou, preferencialmente, num avanço tecnológico, materializado por certo aumento da eficácia dos fatores de produção ou pela disponibilidade de "novos produtos, processos e serviços e, em alguns casos, setores totalmente novos" (Harrison, 2005: 55).

Uma das atividades do gerenciamento tecnológico é a transferência de tecnologia, entendida como o deslocamento do conhecimento tecnológico de um lugar para outro. Envolvendo geralmente atividades de comunicação e interação, tal deslocamento ocorre através de negociações não comerciais ou por meio de transações comerciais.

Voltada para a mudança ou inovação tecnológica, a transferência de tecnologia não se reduz a atividades de venda e compra de materiais, plantas, máquinas e ferramentas. Ela implica também na mobilização de indivíduos e organizações (Cribb, 1999 e 2000). Nonaka (1991: 97) destaca este aspecto, assinalando que "o novo conhecimento começa sempre com o indivíduo".

No processo de transferência de tecnologia, há pelo menos duas partes: a emissora e a receptora de uma determinada tecnologia. Com estatutos e objetivos diferentes, cada uma das partes tem seus próprios interesses que podem até ser dificilmente conciliáveis. $O$ processo de transferência de tecnologia envolve, além desses atores, outros grupos tais como entidades governamentais, usuários finais, instituições internacionais e organizações não governamentais de desenvolvimento (Nelson, 1993; Cribb, 1999).

A transferência de tecnologia não é a imitação passiva de tecnologias oriundas de fontes externas, mas é um processo ativo e criativo de adaptação que leva em conta as condições locais de atuação das partes envolvidas. Os mecanismos de transferência de tecnologia são diversos e

ISSN: 07I 8-2724. (http://www.jotmi.org)

Journal of Technology Management \& Innovation (C) Universidad Alberto Hurtado, Facultad de Economía y Negocios 
incluem $\bigcirc$ licenciamento de tecnologia, acordos cooperativos de P\&D, a assistência técnica, o compartilhamento de uso de equipamentos, programas de troca de tecnologias, publicações e palestras. "Na verdade, a transferência de tecnologia é um processo estrutural de aprendizado" (Sankat et al., 2007: 646).

O aprendizado é um dos mais significativos processos dinâmicos ocorridos nas empresas, no âmbito das quais ele constitui a base da mudança técnica incremental. Graças a ele, se constróem e se consolidam a aquisição, acumulação e geração do conhecimento. Existe uma ampla variedade de processos de aprendizado, tais como "learning by doing, learning by using, learning from advances in science and technology, learning from inter-industry spillovers, learning by interacting e learning by searching" (Malerba, 1992: 849).

O deslocamento de uma tecnologia de uma organização emissora a uma empresa receptora não se faz sem custo (Teece, 1977), pois exige alocação de recursos para a transmissão e absorção do conhecimento tecnológico. $O$ aprendizado não é um bem gratuito, mas sim uma atividade custosa, focalizada e multidimensional. Em razão da diversidade das características e influências dos processos de aprendizado, as empresas podem ter direções diferentes de mudança técnica incremental. Outra observação que merece ser destacada é que o aprendizado não surge do nada. As fontes externas de $P \& D$ desempenham um papel importante na acumulação do estoque de conhecimento das empresas e na geração de trajetórias de avanço técnico incremental (Malerba, 1992).

Geralmente orientado pela busca de inovação, o sucesso da transferência de tecnologia depende significativamente do desempenho de cada uma das partes envolvidas. Segundo Metcalfe (1995: 34),

"o fato central a respeito do processo moderno de inovação é que ele é baseado numa divisão do trabalho, (...). A divisão do trabalho produz eficientemente ganhos a partir da especialização e profissionalização, mas ela requer também um arcabouço para conectar juntamente as contribuições dos diferentes atores".

Nesse sentido, um aspecto fundamental do processo de transferência de tecnologia refere-se à capacidade absortiva da empresa receptora. É essa capacidade que facilita a assimilação dos elementos da tecnologia por parte da empresa. Ela se traduz pela competência de identificar tecnologias disponíveis para aquisição e imitação, modificálas e adaptá-las a necessidades produtivas. A capacidade das empresas de aproveitar de resultados de P\&D constitui um fator fundamental de seu futuro desempenho (Cohen \& Levinthal, 1990; Cribb, 1999; Haro-Domínguez et al., 2007). No setor agroalimentar, as organizações públicas de P\&D, imbuídas da importância de tal capacidade para o processo de transferência tecnológica, se empenham geralmente não apenas na geração de tecnologias, mas também na assistência e capacitação técnica de seus clientes, beneficiários e parceiros (Cribb, 1999).

\section{Metodologia}

A pesquisa da qual surgiu este artigo foi realizada num período de oito anos junto a médias, pequenas e micro empresas agroindustriais ${ }^{5}$, clientes da Embrapa Agroindústria de Alimentos. Suas atividades foram concretizadas conforme um processo iterativo baseado na triangulação de fontes e métodos. Essas podem ser reagrupadas em quatro blocos: revisão de literatura, coleta de informações e dados, observação nas empresas pesquisadas e tratamento das informações e dos dados coletados.

\section{I Revisão de literatura}

Este bloco de atividades foi realizado através do banco de dados da Embrapa Agroindústria de Alimentos e de textos escritos nos últimos 40 anos sobre a transferência de tecnologia e o setor agroindustrial brasileiro. A revisão da literatura ajudou principalmente na compreensão de conceitos-chaves a respeito da transferência de tecnologia e na elaboração da lista das empresas pesquisadas. Também, facilitou a identificação de especialistas do setor agroalimentar brasileiro.

\subsection{Coleta de informações e dados}

As atividades de coleta de informações e dados foram realizadas em três momentos. $O$ primeiro correspondeu a uma etapa do projeto de pesquisa descrito em Cribb (2000) e se estendeu no período de março de $200 \mathrm{I}$ a março de 2002. O segundo momento, considerado etapa

\footnotetext{
${ }^{5}$ A classificação das empresas em médias, pequenas e micro foi obtida do banco de dados da Embrapa Agroindústria de Alimentos.
} 
complementar do projeto, se referiu às ações técnicas de avaliação anual de impactos de tecnologias transferidas pela Embrapa Agroindústria de Alimentos e ocorreu ao longo do período de março 2002 a março 2009. O terceiro momento, definido também como etapa complementar do projeto, foi o de entrevistas com especialistas (pesquisadores e técnicos) do setor agroalimentar e se concretizou no período de março a dezembro de 2006.

No início do primeiro momento da coleta de informações e dados, ou seja, em março de $200 \mathrm{I}$, foram enviados questionários a $6 \mathrm{I}$ empresas que se beneficiaram de tecnologias e serviços gerados pela Embrapa Agroindústria de Alimentos nos anos de 1999 e 2000. Desta forma, tratou-se de entrevistas estruturadas. Até outubro de 200 I, foram retornados sete questionários preenchidos; 0 que correpondeu a uma taxa de resposta de aproximadamente $11 \%$. Esta taxa, considerada baixa, foi o reflexo da pouca tradição do empresariado nacional em conceder dados e informações sobre seus empreendimentos. Tal constatação já foi relatada por Araújo (1996) e Matesco (2000). Por isso, foi decidido dar continuidade ao levantamento de dados e informações em outros períodos. Com a prorrogação de tal levantamento até março de 2002, ○ número de questionários preenchidos passou de 7 para 15. Assim, a taxa de resposta subiu de II\% para $25 \%$.

No segundo momento da coleta de informações e dados, outras dez empresas foram pesquisadas por ocasião da realização das atividades anuais de avaliação dos impactos de tecnologias. Nessas últimas empresas, a coleta de informações e dados foi realizada por meio de entrevistas semi-estruturadas que ajudaram a melhor entender as especificidades do processo de transferência de tecnologia na agroindústria brasileira de alimentos.

Os temas, abordados nesses dois momentos da coleta de informações e dados, foram diversos e incluíram o desempenho e a transparência da Embrapa Agroindústria de Alimentos, $\circ$ interesse em colaboração para financiamento de pesquisa, a aquisição de conhecimentos tecnológicos, a busca de capital de desenvolvimento de negócios, a comunicação empresarial e a cooperação financeira entre concorrentes.

No terceiro momento da coleta de informações e dados, foram empreendidas entrevistas não-estruturadas com 9 especialistas (pesquisadores e técnicos) do setor agroalimentar brasileiro. Esses foram escolhidos em três organizações de pesquisa e extensão. As entrevistas ajudaram a obter esclarecimentos sobre $o$ macroambiente em que se realiza a transferência de tecnologias agroindustriais. Tais esclarecimentos permitiram melhor entender as respostas recebidas junto às empresas agroindustriais por ocasião do primeiro e segundo momento da coleta de informações e dados.

\subsection{Observação nas empresas pesquisadas}

A observação in loco foi concretizada, sobretudo através de visitas e reuniões nas empresas onde foram realizadas as atividades de avaliação anual de impactos de tecnologias, e também em locais onde houve feiras e outros eventos relativos ao setor agroindustrial. Permitiu contatos diretos e frequentes com as realidades agroindustriais. Tais contatos foram importantes para a compreensão da dinâmica do setor agroalimentar.

\subsection{Tratamento das informações e dos dados coletados}

O tratamento das informações e dos dados coletados foi efetuado por meio de métodos descritivos. As ferramentas utilizadas incluíram gráficos de frequência e diagramas de causa-efeito.

\section{Resultados e discussão}

A análise dos dados e informações consistiu na procura de diferenças e similaridades entre fatos revelados pelas empresas agroindustriais, não só interpretando o significado dessas diferenças e similaridades, mas também tentando descobrir regularidades baseadas nelas. Os resultados são abaixo apresentados e discutidos.

\section{I Perfil das empresas informantes}

As funções principais das empresas que forneceram dados e informações foram diversas e consistiram em três categorias: pesquisa e desenvolvimento (P\&D); processamento de produtos; e, empacotamento de produtos agrícolas. $7 \%$ das empresas estão envolvidas na primeira categoria; $66 \%$ na segunda; e, $27 \%$ na terceira. $O$ relativo baixo percentual de empresas agroindustriais privadas tendo P\&D como função principal foi compatível com os indicadores observados, conforme os quais, no 
Brasil, as universidades e os laboratórios públicos são os principais lugares da pesquisa (Cribb, 2007).

Foi anotada uma concentração dos empregados das empresas em atividades de produção de bens. De fato, os empregados foram distribuídos da seguinte maneira: $13 \%$ em administração; $7 \%$ em $\mathrm{P} \& D$; e, $80 \%$ em produção de bens.

Em termos de eficiência produtiva, as categorias de processamento de produtos e empacotamento de produtos agrícolas se encontraram em níveis próximos. A taxa de eficiência, ou seja, a relação entre produção efetiva e capacidade de produção foi, em média, de: $30 \%$ para as empresas de P\&D; 65\% para as empresas de processamento de produtos; e, $70 \%$ para as empresas de empacotamento de produtos agrícolas.

\subsection{Percepções das empresas acerca da Embrapa Agroindústria de Alimentos}

Um conjunto de perguntas foi usado para caracterizar as experiências das empresas com a Embrapa Agroindústria de Alimentos. Estas perguntas apresentaram aos informantes alternativas de respostas para serem escolhidas.

Uma das perguntas foi formulada para conferir se as empresas já haviam usado serviços da Embrapa Agroindústria de Alimentos. Todas as empresas responderam afirmativamente.

Para a avaliação dos serviços da Embrapa Agroindústria de Alimentos, foi definida uma pergunta sugerindo cinco níveis de desempenho: excelente, bom, médio, regular e ruim. Nenhuma empresa achou regular ou ruim a atuação da Embrapa Agroindústria de Alimentos. A Tabela I apresenta as percentagens de empresas que escolheram cada um dos demais níveis. $O$ desempenho da Embrapa Agroindústria de Alimentos é fundamentalmente a expressão da motivação de seus recursos humanos na concretização da transferência tecnológica.

Outra pergunta foi usada para saber se as empresas estavam dispostas a participar do financiamento de atividades de P\&D que poderiam ser executadas pela Embrapa Agroindústria de Alimentos. As respostas encontram-se na Tabela 2.

\section{Tabela 1. Desempenho da Embrapa Agroindústria de Alimentos}

\begin{tabular}{lccccc}
\hline Serviços & Excelente & Bom & Médio & Regular & Ruím \\
\hline 1. Análises & $33 \%$ & $60 \%$ & $7 \%$ & - & - \\
2. Informações técnicas & $13 \%$ & $80 \%$ & $7 \%$ & - & - \\
3. Consultoria e assistência técnica & $20 \%$ & $60 \%$ & $20 \%$ & - & - \\
4. Treinamento & $13 \%$ & $87 \%$ & - & - & - \\
\hline
\end{tabular}

Tabela 2. Interesse em colaboração para financiamento de pesquisa

\begin{tabular}{lccc}
\hline Tipo de colaboração & Sim & Não & Indecisão \\
\hline Participação em financiamento de P\&D na Embrapa & $53 \%$ & $27 \%$ & $20 \%$ \\
\hline
\end{tabular}

A boa imagem da Embrapa Agroindústria de Alimentos diante as empresas agroindustriais é um aspecto importante para o desenvolvimento de redes colaborativas entre os atores participando do processo de transferência de tecnologia. Mas, a vontade de colaboração das empresas não se traduz automaticamente pela propensão de financiar pesquisas.

Não há dúvida de que o envolvimento na pesquisa por meio da colaboração no seu financiamento seja um indicador do interesse da empresa em adquirir tecnologias. Entretanto, não se pode esquecer que as médias, pequenas e micro empresas não têm geralmente condições para colaborar financeiramente em atividades de P\&D (Arrow, 1962). Portanto, é de esperar que a maioria das empresas, clientes da Embrapa Agroindústria de Alimentos, não esteja disposta a participar do financiamento de atividades de P\&D.

\subsection{Relevância de diversos fatores na transferência de tecnologia}

Outro conjunto de perguntas foi estabelecido para determinar a importância de diversos fatores na 
transferência de conhecimentos tecnológicos. Estas perguntas foram formuladas de maneira a fornecer aos informantes um leque de respostas alternativas, incluindo a possibilidade de eles formularem suas próprias observações. Nesse sentido, foram definidos cinco grupos de perguntas.

O primeiro grupo se referiu à aquisição de conhecimento tecnológico. Ele procurou saber se a empresa aceitaria adquirir um determinado conhecimento tecnológico divulgado pela Embrapa Agroindústria de Alimentos, mesmo que: I) este conhecimento não fosse aquele esperado pela empresa para aplicação imediata; 2) a empresa não tivesse mão-de-obra qualificada para colocar em prática este conhecimento; 3) a adaptação deste conhecimento à realidade da empresa fosse complexa; e,
4) a empresa não tivesse capital próprio disponível para colocar em prática este conhecimento. Também, houve espaços para observações adicionais da empresa a respeito deste grupo de perguntas.

Os resultados podem ser observados na Tabela 3. Como pode-se constatar, a maioria das empresas aceitaria adquirir o conhecimento tecnológico apesar da existência das situações problemáticas. Esta constatação se explica pela confiança das empresas na Embrapa Agroindústria de Alimentos. Um dos informantes que escolheram a resposta "não" para a quarta pergunta deste grupo destacou que, pelo fato de sua empresa ser de pequeno porte, precisaria de uma linha de crédito subsidiada para poder investir de forma concreta.

\section{Tabela 3. Aquisição de conhecimentos tecnológicos}

\begin{tabular}{lccc}
\hline Situação problemática & Sim & Não & Indecisão \\
\hline 1. Conhecimento não esperado para aplicação imediata & $60 \%$ & $40 \%$ & - \\
2. Inexistência de mão-de-obra qualificada & $67 \%$ & $33 \%$ & - \\
3. Complexidade de adaptação do conhecimento & $80 \%$ & - & $20 \%$ \\
4. Indisponibilidade do capital próprio para o conhecimento & $53 \%$ & $47 \%$ & - \\
\hline
\end{tabular}

O segundo grupo foi constituído de uma única pergunta formulada acerca da disposição da empresa em procurar financiamento para adquirir um conhecimento tecnológico competitivo e colocá-lo em prática. Também, foram reservados espaços para observações das empresas.

Os resultados são apresentados na Tabela 4. Apesar de declarar sua disposição para tal procura, outro informante (diferente daquele acima citado) observou que a disposição do empreendedor não garantiria nem facilitaria a obtenção dos recursos financeiros necessários. $O$ fato de que todas as empresas têm disposição em procurar financiamento para adquirir o conhecimento tecnológico mostra que a existência da tecnologia e a disponibilidade de capital necessário ao aproveitamento desta são determinantes significativos no processo de transferência tecnológica.

\section{Tabela 4. Busca de capital de desenvolvimento}

\begin{tabular}{lccc}
\hline Razão da busca & Sim & Não & Indecisão \\
\hline Financiamento para aquisição e uso de conhecimento & $100 \%$ & - & - \\
\hline
\end{tabular}

O terceiro grupo foi composto de perguntas formuladas acerca das características socioeconômicas da transmissão de conhecimentos tecnológicos. Foi dividido em dois subgrupos, procurando respectivamente saber: I) que meios permitiram à empresa ter informações sobre serviços e produtos existentes na Embrapa Agroindústria de Alimentos; e, 2) a influência de diversos fatores sobre a aquisição de conhecimentos tecnológicos.

No que diz respeito ao primeiro subgrupo, foram fornecidas algumas alternativas de respostas: jornais, internet e folders.
Também, foram disponibilizados ao informante espaços para inserir outros meios de informação. Dentro da categoria de outros meios de informação, os contatos pessoais foram objeto de mais respostas.

No caso do segundo subgrupo, foram apresentados quatro fatores (prazo de atendimento, preço do serviço fornecido e qualidade do serviço) cuja influência respectiva sobre a aquisição de conhecimentos tecnológicos foi avaliada pelas empresas. A qualidade do serviço, aspecto fundamental para a caracterização de uma organização de P\&D, foi 
definida como um fator baseado nos seguintes critérios: atendimento dentro do prazo previsto e viabilidade dos prazos com relação à urgência das necessidades de aquisição do conhecimento tecnológico. Ainda neste subgrupo, foram sugeridos cinco níveis de influência: muito alto, alto, médio, baixo e nenhum. Os dois últimos não foram escolhidos por nenhuma empresa.

Os resultados dos dois subgrupos encontram-se respectivamente nas Tabelas 5 e 6 .

Tabela 5. Meios de informações

\begin{tabular}{lc}
\hline Meios de informação & Números de respostas \\
\hline 1. Jornais & 9 \\
2. Folders & 6 \\
3. Internet & 4 \\
4. Outros & 11 \\
\hline
\end{tabular}

Tabela 6. Influência de fatores sobre aquisição de conhecimento

\begin{tabular}{lccccc}
\hline Fatores & Muito alta & Alta & Média & Baixa & Nenhuma \\
\hline 1. Prazo de atendimento & $27 \%$ & $27 \%$ & $46 \%$ & - & - \\
2. Preço & $60 \%$ & $27 \%$ & $13 \%$ & - & - \\
3. Qualidade & $66 \%$ & $27 \%$ & $7 \%$ & - & - \\
\hline
\end{tabular}

O quarto grupo abrangeu duas perguntas usadas para conhecer as percepções das empresas agroindustriais sobre condições de transparência da Embrapa Agroindústria de Alimentos. A primeira foi uma pergunta para saber se a Embrapa Agroindústria de Alimentos precisava intensificar mais seus programas de comunicação com as empresas. A segunda foi formulada para saber se, no planejamento das atividades da Embrapa Agroindústria de Alimentos, a participação das empresas era necessária. Os resultados são apresentados na Tabela 7 .

Tabela 7. Transparência na Embrapa Agroindústria de Alimentos

\begin{tabular}{lccc}
\hline Ação necessária & Sim & Não & Indecisão \\
\hline 1. Mais comunicação & $93 \%$ & - & $7 \%$ \\
2. Mais participação dos clientes & $87 \%$ & - & $13 \%$ \\
\hline
\end{tabular}

○ fato de uma tecnologia existir não significa que sua completa transferência seja garantida. Como afirmam Dorf \& Worthington (1990: 265), "geralmente, as empresas reconhecem o valor de novas descobertas realizadas em universidades ou laboratórios públicos, mas algumas complicações acerca da comunicação e segurança, assim como dos arranjos contratuais, tendem a torná-las não completamente motivadas".

O quinto grupo também consistiu em duas perguntas usadas para saber como as empresas visualizavam a participação ou colaboração de outros atores na rede de relações necessárias à transferência de conhecimentos tecnológicos. A primeira pergunta se referiu à indicação das três principais ações que o setor público (governo federal, estadual e/ou municipal) podia empreender para melhorar o processo de aquisição de conhecimentos tecnológicos. Responderam a esta pergunta 60\% dos informantes. As respostas foram diversas e incluíram principalmente propostas tais como: isenção de impostos; parcerias financeiras; parcerias visando aumento de mão de obra; melhoria na comunicação entre a Embrapa Agroindústria de Alimentos e a empresa; promoção do intercâmbio entre organizações de pesquisa e unidades produtivas via redução de preços de acesso a serviços. Os informantes justificaram essas propostas pelo fato de que as atividades agroindustriais dependem amplamente de fatores climáticos e apresentam um carater sazonal. Apesar de sua confiança na competência da Embrapa Agroindústria de Alimentos como organização de P\&D e de sua disposição em procurar financiamento, eles destacaram a necessidade da adequação contínua dos mecanismos de agilização do setor agroindustrial brasileiro. A segunda pergunta foi formulada para saber a disposição 
da empresa em financiar, junto com empresas concorrentes, uma atividade voltada ao desenvolvimento ou aquisição de um novo conhecimento tecnológico. As respostas estão na Tabela 8.

Tabela 8. Possibilidade de cooperação financeira com concorrentes

\begin{tabular}{lccc}
\hline Tipo de cooperação & Sim & Não & Indecisão \\
\hline Cooperação para P\&D & $27 \%$ & $53 \%$ & $20 \%$ \\
\hline
\end{tabular}

Graças a sua boa imagem, a Embrapa Agroindústria de Alimentos ganha a confiança das empresas agroindustriais privadas. Mas, esta confiança não é suficiente para assegurar a transferência ou a ampla difusão do conhecimento tecnológico. É preciso ter recursos financeiros para poder investir não apenas em capacidade absortiva, mas também em infra-estrutura tecnológica (Cribb, 1999). Nesse sentido, a implantação de uma política tecnológica apropriada, associada à política industrial, pode ter efeitos significativamente positivos sobre o processo de transferência de tecnologia. Além de uma política tecnológica, a interação entre a emissora e a receptora da tecnologia precisa ser continuamente fortalecida através da adequação sempre renovada dos meios de comunicação, da multiplicação dos canais de participação e da melhoria das condições de disponibilização de produtos e serviços (Lin, 2003). A cooperação entre empresas concorrentes para desenvolvimento ou aquisição de conhecimentos tecnológicos merece ser incentivada, sobretudo nos casos de tecnologias pré-competitivas.

\section{Conclusão}

Pelos resultados, os determinantes da transferência de tecnologia na agroindústria brasileira são múltiplos. Eles incluem a imagem da organização de P\&D, a disponibilidade da tecnologia transferível, a informação sobre as características da tecnologia gerada, o capital necessário ao aproveitamento da tecnologia, o envolvimento do usuário potencial da tecnologia no projeto de pesquisa, o interesse por parte do usuário potencial e a motivação do pesquisador na transferência da tecnologia.

A Embrapa Agroindústria de Alimentos tem construído sua imagem baseando-se essencialmente no seu desempenho. Graças à qualidade dos produtos fornecidos e serviços prestados, ela tem adquirido e consolidado sua legitimidade, credibilidade e pertinência. Sua capacidade em responder às expectativas agroindustriais foi favoravelmente reconhecida pelas empresas pesquisadas.
Tal reconhecimento tem sido fundamental na aquisição e adoção de novos conhecimentos tecnológicos por parte da maioria destas que, mesmo em situações produtivas problemáticas, não hesitariam em adquirir produtos e serviços da Embrapa Agroindústria de Alimentos.

A existência da tecnologia a ser transferida tem sido também de fundamental importância. Tanto as características quanto as funções da tecnologia têm sido ingredientes utilizados pelas empresas agroindustriais no processo de adoção tecnológica.

A informação, determinante mais destacado em todas as etapas da pesquisa, aparece como um fator bastante importante na tomada de decisão das empresas agroindustriais a respeito de uma nova tecnologia. Ela tem sido procurada por estas em diversos meios de divulgação técnica, dentre os quais destacam-se jornais, folders e internet. A grande maioria das empresas (93\%) destacou a necessidade de mais comunicação direta entre elas e a Embrapa Agroindústria de Alimentos.

O capital foi visto por todas as empresas pesquisadas como um fator que influencia bastante sua decisão. Pode ser próprio ou emprestado. Mas, para elas, o importante é tê-lo no momento certo para tirar proveito de uma tecnologia recomendada pela Embrapa Agroindústria de Alimentos.

O envolvimento do usuário potencial nas atividades de P\&D foi reconhecido como necessário para desenvolver o entrosamento entre o emissor e o receptor da tecnologia esperada. A maioria das empresas (87\%) quer mais participação nessas atividades. Tal envolvimento pode, em caso de geração e transferência da tecnologia, facilitar a adoção e assimilação desta pelo usuário.

O interesse do usuário potencial na tecnologia foi também levantado como determinante importante. Em termos de financiamento de pesquisa, as respostas obtidas mostraram que ele tem sido de caráter mais individual do que 
coletivo. Enquanto mais da metade das empresas (53\%) manifestou seu desejo de contribuir em atividades de P\&D, apenas $27 \%$ se sentiram dispostas a cooperar com seus concorrentes para desenvolver ou adquirir novos conhecimentos tecnológicos.

A motivação do pesquisador na transferência de tecnologia foi também abordada pelas empresas pesquisadas. A expressiva maioria dessas empresas $(80-100 \%)$ achou excelentes ou bons os serviços prestados pelos profissionais da Embrapa Agroindústria de Alimentos. Graças aos comportamentos desses profissionais, as empresas têm tido percepções satisfatórias acerca da Embrapa Agroindústria de Alimentos.

Ao identificar e caracterizar determinantes da transferência de tecnologia na agroindústria de alimentos, este artigo apresenta uma base para estudos e práticas referentes à mudança tecnológica no setor produtivo. Os diferentes atores do setor agroalimentar - tais como entidades governamentais, agências de crédito e instituições de fomento de P\&D - tendo conhecimento desses determinantes, podem melhor definir suas estratégias para alcançar seus objetivos. Espera-se que os resultados, apresentados e analisados neste artigo, possam auxiliar na definição ou reformulação de políticas públicas favoráveis à geração e aplicação de tecnologias na agroindústria brasileira de alimentos.

\section{Referências}

ARAÚJO, V. M. R. H. de. (1996) Informação e assistência técnica à agroindústria. Rio de Janeiro, EMBRAPA / IICA.

ARROW, K. J. (1962) Economic welfare and the allocation of resources for invention. Pp. 609-625. In: Nelson, R. R. (ed.) The Rate and Direction of Inventive Activity. New York, Princeton University Press.

AUSTIN, J. E. (198I) L'analyse des projets agro-industriels. Paris: Economica, 2I2p.

AUTIO, E., Laament, T. (1995) Measurement and evaluation of technology transfer: review of technology transfer mechanisms and indicators. International Journal Technology Management, vol. 10, $n^{\circ}$ 7/8, pp. 643-664.
BARROS, J. R. M., Barros, A L. M. (2205) A geração de conhecimento e o sucesso do agronegócio brasileiro. Revista de Política Agrícola, v. I4, n. 4, Pp. 5-I4.

BURGELMAN, R. A., Christensen, C. H., Wheelwright, S. C. (2004) Strategic management of technology and innovation. $4^{\text {a }}$ Ed. Boston, McGraw Hill,.

COHEN, W. L., Levinthal, D. A. (1990) Absortive capacity: a new perspective on learning and innovation. Administrative Sciences Quarterly, vol.35, n I, pp. I28-152.

CRIBB, A. Y. (1999) Acumulação de capacidades biotecnológicas no sistema alimentar: uma matriz de estratégias para países em desenvolvimento. 285p. Tese (Doutorado em Engenharia de Produção). Coordenação dos Programas de Pós-Graduação de Engenharia, Universidade Federal do Rio de Janeiro, Rio de Janeiro.

CRIBB, A. Y. (2000) Transferência de conhecimentos tecnológicos: o caso da Embrapa Agroindústria de Alimentos. Rio de Janeiro. 22 p. (CNPq 300057/00-8 NV). Projeto concluído.

CRIBB, A. Y. (2004) Sistema agroalimentar brasileiro e biotecnologia moderna: oportunidades e perspectivas. Cadernos de Ciência \& Tecnologia, Brasília, v.2I, n. I, pp. 169-195.

CRIBB, A. Y. (27 nov. 2007) Mudança cultural coletiva: o pré-requisito da inovação no Brasil. Jornal da Ciência, Rio de Janeiro, RJ, p. I - 2, http://www.jornaldaciencia.org.br/ Detalhe.jsp?id=52580 (Acesso em 18 maio 2009).

CRIBB, A. Y., Cribb, S. L. de S. P., Silva, F. T. (2008) Organização cooperativista e adoção tecnológica: um estudo de caso na agricultura familiar. In: Encontro de pesquisadores latino-americanos de cooperativismo, 5. Ribeirão Preto: FEARP/USP, I CD-ROM.

DORF, R. C., Worthington, K. K. F. (1990) Technology transfer from universities and research laboratories. Technological forecasting and social change, vol. 37, $n^{\circ} 3$, pp. 25I-266.

DOSI, G. (1988) Sources, procedures and microeconomic effects of innovation. Journal of Economic Literature, vol. 26, $n^{\circ}$ 3, pp.II20-II7I. 
EMBRAPA Agroindústria de Alimentos. (2009) Embrapa Agroindústria de Alimentos transformando tecnologia em soluções. Rio de Janeiro: Embrapa Agroindústria de Alimentos. http://www.ctaa.embrapa.brl. (Acesso em 17 maio 2009).

FARINA, E. (200I) Challenges for Brazil's food industry in the context of globalization and Mercosur consolidation. International food and Agribusiness Management review, Vol. 2, $\mathrm{n}^{\circ} 3$ / 4, pp. 315-330.

GASQUES, J. G., Bastos, E. T.; Bacchi, M. P. R.; Conceição, J. C. P. R. (2004) Condicionantes da produtividade da agropecuária brasileira. Texto para discussão, $\mathrm{n}^{\circ} 1017,33 \mathrm{p}$. Brasília: IPEA.

GILBERT, M., Cordey-Hayes, M. (1996) Understanding the process of knowledge transfer to achieve successful technological innovation. Technovation, vol.16, $n^{\circ} 6$, pp.30I312.

GOODMAN, D., Sorj, B., Wilkinson, J. (1990) Da lavoura às biotecnologias: agricultura $\mathrm{e}$ indústria no sistema internacional. Rio de Janeiro, Campus. 192p.

GUAN, J. C., Mok, C. K., Yam, R. C. M., Chin, K. S., Pun, K. F. (2206) Technology transfer and innovation performance: Evidence from Chinese firms. Technological Forecasting \& Social Change, vol. 73, $n^{\circ}$ 6, pp. 666-678.

GUELLEC, D. (coord.). (1993) Innovation et compétitivité. Paris, Economica. 246p.

HARO-DOMÍNGUEZ, M. C., Arias-Aranda, D., LlorénsMontes, F. J., Moreno, A. R. (2007) The impact of absorptive capacity on technological acquisitions engineering consulting companies. Technovation, vol. $27, \mathrm{n}^{\circ}$ 8, pp. 4I7-425.

HARRISON, J. S. (2005) Administração estratégica de recursos e relacionamentos. Trad. Luciana de Oliveira da Rocha. Porto Alegre, Bookman.

JANK, M. S., Nassar, A. M. (2000) Competitividade e globalização. In: ZYLBERSZTAJN, D.; NEVES, M. F. (Org.). Economia e gestão dos negócios alimentares: indústria de alimentos, indústria de insumos, produção agropecuária, distribuição. São Paulo, Pioneira. cap. 7, pp. I37-163.
JANK, M. S., Nassar, A. M., Tachinardi, M. H. (2005) Brasil, potência agricola mundial. http://www.granos.agr.br/ stored/I202821722 85335.pdf (Acesso em 18 maio 2009).

LIN, B.-W. (2003) Technology transfer as technological learning: a source of competitive advantage for firms with limited R\&D resources. R\&D Management, vol. $33, n^{\circ} 3$, PP. 327-34I.

MALERBA, F. (1992) Learning by firms and incremental technical change. The Economic Journal, vol. 102, $n^{\circ} 413$, pp. 845-859.

MATESCO, V. R. (2000) Comportamento tecnológico das empresas transnacionais em operação no Brasil. São Paulo, SOBEET.

METCALFE, J. S. (1995) Technology systems and technology policy in an evolutionary framework. Cambridge Journal of Economics, vol. 19, $\mathrm{n}^{\circ}$ I, pp. 25-46.

NEFUSSI, J. (1989) Les industries agro-alimentaires. Paris, PUF. 128p.

NELSON, R. R. (org.). (1993) National innovation systems: a comparative analysis. New York, Oxford University Press.

NONAKA, I. (199I) The knowledge-creating company, Havard Business Review, Nov.-Dez., pp. 96-104.

NUNES, E. P., Contini, E. (2000) Dimensão do complexo agroindustrial brasileiro. São Paulo, ABAG.

PINTO, L. C. G. (2006) O crescimento recente do agronegócio brasileiro. Revista de política agrícola, vol. 15, $\mathrm{n}^{\circ}$ 3, pp. 3 e 4, Jul./Ago./Set. 2006. [Carta da Agricultura].

REARDON, T., Barrett, C. B. (2000) Agroindustrialization, globalization, and international development: an overview of issues, patterns, and determinants. Agricultural Economics, vol. 23, n 3, pp. 195-205.

RIPA - Rede de Inovação e Prospecção tecnológica para o Agronegócio. (2008) Cenários do Ambiente de Atuação das Instituições Públicas e Privadas de PD\&I para o Agronegócio e o Desenvolvimento Rural Sustentável - Horizonte 2023. São Carlos: RIPA/CTAgro. 98p. 
ROSENBERG, N. (1982) Inside the black box: technology and economics. Cambridge: Cambridge University Press. 322p.

SANKAT, C. K., Pun, K. F., Motilal, C. B. (2007) Technology transfer for agro-industries in developing nations: a Caribbean perspective. International Journal Agricultural Resources, Governance and Ecology, vol. 6, $n^{\circ} 6$, pp. 642-665.

TEECE, D. J. (1977) Technology transfer by multinational firms: the resource cost of transferring technological know-how. The Economic Journal, vol. 87, n 346, pp. $242-$ $26 I$.

UNCTAD - United Nations Conference on Trade and Development. (1996) Technology, competitiveness, and industrial policies. Cap. III, pp.53-99. In: ---------. Fostering technological dynamism: evolution of thought on technological development processes and competitiveness: a review of the literature. United Nations Conference on Trade and Development. New York, United Nations.

WANG, T. Y., Chien, S. C. (2007) The influences of technology development on economic performance - The example of ASEAN countries. Technovation, vol. 27, $n^{\circ} 8$, PP. $47 \mathrm{I}-488$.

\section{Sobre o autor}

André Yves Cribb. D.Sc. em Engenharia de Produção pelo Instituto Alberto Luiz Coimbra de Pós-Graduação e Pesquisa de Engenharia da Universidade Federal do Rio de Janeiro (COPPE/UFRJ). Pesquisador da Área de Comunicação e Negócios da Embrapa Agroindústria de Alimentos (CEP 23020-470 - Rio de Janeiro/RJ, Brasil). 